\title{
Evaluation of maternal thyroid autoimmune status in gestational trophoblastic disorders
}

\author{
Erbil Karaman”, Orkun Çetin, Numan Çim, Ali Kolusarı, Seyithan Güler, Recep Yıldızhan, İsmet \\ Alkış, Hanım Güler Şahin
}

Department of Obstetric and Gynecology, Yuzuncu Yil University Medical Faculty, Van, Turkey

\begin{abstract}
To investigate the maternal autoimmune status of patients with molar pregnancies.

The study was conducted at Yuzuncu Yil University, Department of Obstetrics and Gynecology between January 2012 and June 2016. The medical records of patients who were diagnosed and followed for complete and partial molar pregnancies were reviewed retrospectively. The data of patients who were studied for maternal thyroid auto-antibodies including AntiThyroid peroxidase (TPO) and Anti-Tyroglobulin (Tg) were included in the study.

The mean maternal age of complete molar pregnancies were significantly higher than partial molar pregnancies $(p=0.010)$. There were no significant differences between two groups in terms of beta-hCG, TSH, and free T4 $(p=0.815, p=0.204$, and $\mathrm{p}=0.072$, respectively). None of the patients were anti-TPO positive in the study. There was no significant difference in anti-thyroglobulin positivity between the two groups $(\mathrm{p}=0.950)$.

Maternal thyroid autoantibody status did not show any difference between the partial and complete molar pregnancies. Thyroid dysfunction seen in molar pregnancies seems to be due to the stimulation of follicular cell receptors with hCG rather than auto-antibodies against thyroid gland.
\end{abstract}

Key Words: Molar pregnancy, thyroid gland, autoantibody, thyroid peroxidase, tyroglobulin

\section{Introduction}

Hyperthyroidism or increased thyroid function has been reported in many patients with trophoblastic tumors, either hydatidiform mole or choriocarcinoma (1). In the US, hydatidiform mole occurs between 0.5 and 2.5 per 1000 pregnancies (2). It is more common in Asian and Latin American countries (3). Hydatidiform moles secrete large amounts of human chorionic gonadotropin (hCG), and the hCG level is proportional to the mass of the tumor (3). The prevalence of increased thyroid function in patients with hydatidiform mole has been reported as $25-64 \%$. About $5 \%$ have clinical hyperthyroidism (4).

On the other hand, around $0.3-0.5 \%$ of all pregnant women will have overt hypothyroidism $(\mathrm{OH})$ that is associated with an increased risk for adverse pregnancy complications such as premature delivery, low birth weight, miscarriage, as well as detrimental effects on fetal neurocognitive development when untreated. Thyroid autoimmunity and subclinical hypothyroidism are more common than $\mathrm{OH}$, affecting $11 \%$ and $2-2.5 \%$ of reproductive aged women, respectively (5). Thyroid peroxidase (TPO) is a key enzyme in the production of thyroid hormones, and a major autoantigen in autoimmune thyroid diseases (6). Thyroglobulin $(\mathrm{Tg})$ (also called thyroid antibody 1 ) is a dimeric protein produced by the follicular cells of the thyroid (7). Both TPO and Tg are essential in the synthesis and secretion of thyroid hormones, including thyroxine (T4) and triiodothyronine (T3) (7). Autoantibodies to TPO (anti-TPO) and Tg (anti-Tg) are considered secondary responses and indicative of thyroid inflammation, and can be detected in $10-20 \%$ of women at childbearing age (8). Production of the above autoantibodies implicates the activation of an autoimmune process in the thyroid gland, but the majority of women with positive thyroid autoantibodies are euthyroid, that is, they have normal thyroid function.

The association between gestational trophoblastic diseases and maternal thyroid functions was well established in previous studies. However, there was a lack of information about the relation between thyroid autoimmunity and molar pregnancies. In the present study, we aimed to investigate the maternal thyroid autoimmune status of molar pregnancies. We also compared the maternal thyroid autoantibody positivity of complete and partial molar pregnancies. 


\section{Material and methods}

This single center, case-control, retrospective study was conducted at Yuzuncu Yil University Medical Faculty, Obstetrics and Gynecology clinic in Van, Turkey. This study was approved by the institutional ethics committee of Yuzuncu Yil University. We reviewed the medical records of 71 molar pregnancies between January 2012 and June 2016 in our tertiary referral center. Among them, 32 patients who were initially screened for thyroid auto-antibodies were selected to the study population. Exclusion criteria were obvious symptoms of hyperthyroidism or hypothyroidism, a history of treatment with thyroid hormones or antithyroid drugs, a history of autoimmune diseases, congenital heart diseases or heart failure and increased liver enzymes or serum creatinine. These patients were divided into two groups according to pathological findings. The first group comprised 12 patients with complete molar pregnancies. The second group comprised 20 patients with partial molar pregnancies. Diagnosis of gestational trophoblastic disease was confirmed by histopathological examination of endometrial curettage specimens. Molar pregnancy cases were managed by suction evacuation or by hysterectomy in multiparous women above 40 years of age. The initial beta-hCG, thyroid stimulating hormone (TSH), free $\mathrm{T} 4$, $\mathrm{Tg}$, antiTPO and anti-Tg levels were reviewed from the hospital's medical records.

TSH and free T4 values were $0.03-2.3 \mu \mathrm{U} / \mathrm{ml}$ and 0.86-1.77 $\mathrm{ng} / \mathrm{dl}$, respectively, for the first trimester of pregnancy (9). Thyroglobulin $>60$ $\mathrm{ng} / \mathrm{ml}$, anti-TPO $>5.6 \mathrm{IU} / \mathrm{ml}$ and anti-Tg $>4.1$ $\mathrm{IU} / \mathrm{ml}$ were considered positive.

Descriptive statistics for studied variables (characteristics) were presented as Mean, Standard Deviation, Minimum and Maximum values.
Continuous variables were compared among the two groups using Mann-Whitney U test. Fisherexact test was used to compare categorical variables. Statistical significance levels were considered as $5 \%$. The SPSS (IBM SPSS Statistics for Windows, Version 22.0. IBM Corp. Released 2013. Armonk, NY: IBM Corp.) statistical program was used for all statistical computations.

\section{Results}

The clinical characteristics, biochemical analyses and thyroid autoantibody status of the patients were summarized in Table 1 . The mean maternal age of all patients was $37.3 \pm 11.8$ years in the study. The mean maternal age of complete mole pregnancies were significantly higher than partial mole pregnancies $(p=0.010)$. There were no significant differences between two groups with respect to beta-HCG, TSH and free T4 $(\mathrm{p}=0.815$, $\mathrm{p}=0.204$, and 0.072 , respectively). None of the patients were anti-TPO positive in the study. 15 patients were thyroglobulin positive $(15 / 22$, $68.2 \%)$ and 7 patients were anti-Tg positive $(7 / 22$, $31.2 \%$ in the study. Thyroglobulin positivity was more prevalent in complete mole pregnancies than in partial mole pregnancies $(\mathrm{p}=0.003)$. There was no significant difference in anti-thyroglobulin positivity between two groups $(\mathrm{p}=0.950)($ Table 1$)$.

\section{Discussion}

Gestational trophoblastic disorders are neoplastic diseases of the trophoblastic epithelium of the placenta and is classified into four groups: hydatidiform mole (complete or partial), persistent invasive gestation trophoblastic neoplasia, choriocarcinoma, and placental site trophoblastic tumors (10). It is a well-established data that thyroid dysfunctions are more common finding in

Table 1. The demographic and laboratory parameters of the two groups

\begin{tabular}{llll}
\hline & Complete molar pregnancies (n: 12) & Partial molar pregnancies (n: 20) & $\mathrm{p}$ \\
\hline Maternal age & $44.2 \pm 11.4$ & $33.1 \pm 10.2$ & $0.010^{*}$ \\
\hline Beta-hCG & $454879 \pm 393606$ & $542510 \pm 605871$ & $0.815^{*}$ \\
\hline TSH & $0.2 \pm 0.4$ & $0.8 \pm 1.3$ & $0.204^{*}$ \\
\hline Free T4 & $3.6 \pm 5.2$ & $1.6 \pm 0.7$ & $0.072^{*}$ \\
\hline Thyroglobulin $(+)$ & $10(83.3 \%)$ & $5(25 \%)$ & $0.003^{\#}$ \\
\hline Anti-TPO $(+)$ & 0 & 0 & \\
\hline Anti-thyroglobulin $(+)$ & $3(25 \%)$ & $4(20 \%)$ & $0.950^{\#}$ \\
\hline
\end{tabular}

${ }^{*}$ Mann-Whitney U test was used to compare continuous variables.

\# Fischer-exact test was used to compare categorical variables.

hCG, human Chorionic Gonadotropin; TSH, Thyroid Stimulating Hormone; T4, Thyroxine; TPO, Thyroid peroxidase.

East J Med Volume:21, Number:2, April-June/2016 
the molar pregnancies. This association comes from the thought that hCG produced in the women with molar pregnancies enhances the thyrotrophic activity by acting on the thyroid TSH receptors and inducing the release of thyroid hormones (11). In this present retrospective study, we aimed to evaluate the maternal thyroid autoantibody status including anti-TPO and anti$\mathrm{Tg}$ in complete and partial molar pregnancies and to investigate if these autoantibodies have an effect on the thyroid dysfunctions in molar pregnancies. Our study showed that no statistical significance was found between molar pregnancies with regards to the thyroid auto-antibody levels.

However, the thyroid dysfunction seen in molar pregnancies is known to be due to the increased level of hCG, the autoimmune thyroid diseases (AITD) may contribute to this disorder. AITD result from a dysregulation of the immune system leading to an immune attack on the thyroid gland. AITD are $\mathrm{T}$ cell-mediated organ-specific autoimmune disorders. The most important thyroid auto-antibodies are anti-TPO and anti-Tg antibodies. TPO is a crucial enzyme in the thyroid hormone production and $\mathrm{Tg}$ is a dimeric protein produced by the follicular cells of the thyroid gland during production of hormones $(6,7)$. Autoantibodies to TPO and $\mathrm{Tg}$ are known to be as a secondary response and indicative of thyroid inflammation, and it is reported that these can be detected in $10-20 \%$ of women at childbearing age (8). The autoimmune process and effect on thyroid gland can be implicated with the increased level of the above autoantibodies (12). To date, there is no study reporting the evaluation of thyroid auto-antibody levels in molar pregnancies. Our findings suggest that no role of anti-TPO and anti- $\mathrm{Tg}$ autoantibodies have been observed in the thyroid dysfuntion of molar pregnancies.

Although, the thyroid dysfunction in women of reproductive age period due to autoimmunity is a common problem, it is also an important finding during pregnancy. It has been reported that thyroid autoantibodies that included anti-TPO and anti-Tg were found in $6-18 \%$ and $6-16 \%$ of pregnancies, respectively (13). The impact of autoantibodies on the pregnancy has been studied in several reports and it has been found to be associated with recurrent miscarriage, abortion, maternal postpartum thyroid dysfunction neuropsychological impairments and fetal death (14).

The observational studies showed that thyroid autoantibodies even with normal thyroid function are associated with adverse pregnancy outcomes
(14). These effects can be explained by the occurence if subclinical or overt hypothroidism in some euthyroid women with thyroid autoimmunity (15). The routine autoantibody level during pregnancy is not reccomended and there is a lack of data regarding this issue in the literature. In literature search, we found a study that reported by Shaarawy and colleaque studied on the tumor suppresor gene P53 autoantibodies in patients with gestational trophoblastic disorders (16). They reported that a significant positive correlation between P53 autoantibodies and serum hCG $\beta$ concentrations in gestational trophoblastic tumors was observed (16). Based on the current literature, there is a lack of data regarding different auto-antibody levels in patients with gestational trophoblastic diseases.

There are some limitations of our study. First, we evaluated a limited number of patients, the relatively small sample size limits the statistical power of the analysis, to some extent. Secondly, we did not have the opportunity to evaluate thyroid gland of all patients with imaging studies including ultrasound examination. And also, the gestation dependent loss of thyroid autoantibody positivity may limit to reach a more comprehensive data presentation.

In conclusion, gestational trophoblastic diseases including complete and partial molar pregnancies complicates both the physiology of thyroid glands and alters the level of thyroid hormone levels. However, maternal thyroid autoantibody status did not show any difference in partial and complete molar pregnancies. And also, anti-TPO antibody was not observed in molar pregnancies. Our study findings suggest and confirm that the thyroid dysfunction seen in molar pregnancies is due to the stimulation of follicular cell receptors with hCG rather than auto-antibodies against thyroid gland.

\section{References}

1. Yoshimura M, Pekary AE, Pang XP, et al. Thyrotropic activity of basic isoelectric forms of human chorionic gonadotropin extracted from hydatidiform mole tissues. Journal of Clinical Endocrinology and Metabolism 1994; 78: 862864.

2. Palmer JR. Advances in the epidemiology of gestational trophoblastic disease. The Journal of Reproductive Medicine 1994; 39: 155-162.

3. Hershman JM. Physiological and pathological aspects of the effect of human chorionic 
gonadotropin on the thyroid. Best Pract Res Clin Endocrinol Metab 2004; 18: 249-265.

4. Talbot JA, Lambert A, Anobile CJ, et al. The nature of human chorionic gonadotrophin glycoforms in gestational thyrotoxicosis. Clinical Endocrinology (Oxford) 2001; 55: 33-39.

5. Ashoor G, Maiz N, Rotas M, Jawdat F, Nicolaides KH. Maternal thyroid function at 11-13 weeks of gestation and spontaneous preterm delivery. Obstetrics and Gynecology 2011; 117: 293-298.

6. Ruf J, Carayon P. Structural and functional aspects of thyroid peroxidase. Arch. Biochem. Biophy 2006; 445: 269-277.

7. Carayanniotis G. The cryptic self in thyroid autoimmunity: the paradigm of thyroglobulin. Autoimmunity 2003; 36: 423-428.

8. Prummel MF, Wiersinga WM. Thyroid peroxidase autoantibodies in euthyroid subjects. Best Pract. Res. Clin. Endocrinol. Metab 2005; 19: 1-15

9. Panesar NS, Li CY, Rogers MS. Reference intervals for thyroid hormones in pregnant Chinese women. Ann Clin Biochem 2001; 38: 329-332.
10. Smith HO. Gestational trophoblastic disease epidemiology and trends. Clin Obstet Gynecol 2003; 46: 541-556.

11. Tomer Y, Huber GK, Davies TF. Human chorionic gonadotropin (hCG) interacts directly with recombinant human TSH receptors. J Clin Endocrinol Metab 1992; 74: 1477-1479.

12. Stagnaro-Green A, Pearce E. Thyroid disorders in pregnancy. Nat Rev Endocrinol 2012; 8: 650658.

13. Lata K, Dutta P, Sridhar S, et al. Thyroid autoimmunity and obstetric outcomes in women with recurrent miscarriage: a casecontrol study. Endocr Connect 2013; 2: 118-124.

14. Negro R, Mestman JH. Thyroid disease in pregnancy. Best Pract Res Clin Endocrinol Metab 2011; 25: 927-943.

15. Banerjee S. Thyroid disorders in pregnancy. Journal of the Association of Physicians of India 2011; 59: 32-34.

16. Shaarawy M, Sheiba M. Diagnostic and prognostic significance of circulating tumor suppressor gene p53 autoantibodies in patients with gestational trophoblastic tumors. Acta Oncol 2004; 43: 43-48. 